\title{
1 Mapping migration in a songbird using high-resolution genetic markers
}

2

3 Kristen Ruegg ${ }^{1,2}$, Eric C. Anderson ${ }^{3,4}$, Kristina L. Paxton ${ }^{5.6}$, Vanessa Apkenas ${ }^{3}$, Sirena

4 Lao $^{1}$, Rodney B. Siegel ${ }^{7}$, David F. DeSante ${ }^{7}$, Frank Moore ${ }^{6}$ and Thomas B. Smith ${ }^{1,8}$

5

7

$8{ }^{1}$ Center for Tropical Research, Institute of the Environment and Sustainability, University

9 of California, Los Angeles, La Kretz Hall, Suite 300, 619 Charles E. Young Dr. East, Los

10 Angeles, CA 90095, USA

11

$12{ }^{2}$ Department of Ecology and Evolutionary Biology, University of California, Santa Cruz,

13 Santa Cruz, CA 95060, USA; e-mail: kruegg@ ucsc.edu; phone: 510-292-5099

$15{ }^{3}$ Southwest Fisheries Science Center, National Marine Fisheries Service, 110 Shaffer

16 Road, Santa Cruz, CA 95060, USA

17

$18{ }^{4}$ Department of Applied Mathematics and Statistics, University of California, Santa Cruz,

19 CA 95060, USA

20

$21{ }^{5}$ Department of Biological Sciences, University of Southern Mississippi, Hattiesburg,

22 MS 39406

23

${ }^{6}$ Department of Biology, University of Hawaii, Hilo, HI 96720, USA

25

$26{ }^{7}$ The Institute for Bird Populations, PO Box 1346, Point Reyes Station, CA 94956, USA

27

$28{ }^{8}$ Department of Ecology and Evolutionary Biology, University of California, Los

29 Angles, CA, 90095, USA 
31 Neotropical migratory birds are declining across the Western Hemisphere, but

32 conservation efforts have been hampered by the inability to assess where migrants

33 are most limited - the breeding grounds, migratory stopover sites, or wintering

34 areas. A major challenge has been the lack of an efficient, reliable, and broadly

35 applicable method for connecting populations across the annual cycle. Here we

36 show how high-resolution genetic markers can be used to identify populations of a

37 migratory bird, the Wilson's warbler (Cardellina pusilla), at fine enough spatial

38 scales to facilitate assessing regional drivers of demographic trends. By screening

391626 samples using 96 single nucleotide polymorphisms (SNPs) selected from a large

40 pool of candidates $(\mathbf{4 5 0 , 0 0 0})$, we identify novel region-specific migratory routes and

41 timetables of migration along the Pacific Flyway. Our results illustrate that high-

42 resolution genetic markers are more reliable, accurate, and amenable to high

43 throughput screening than previously described tracking techniques, making them

44 broadly applicable to large-scale monitoring and conservation of migratory

45 organisms.

47 Introduction

48 Over half of the Neotropical migrant bird species found breeding in North America have

49 shown marked declines in abundance over the last several decades (Robbins 1989; Sauer

50 et al. 2012). Population declines are thought to relate to stressors encountered by

51 migrants at each stage in the annual cycle - the breeding grounds, the wintering grounds,

52 and migratory stopover points (Rappole 1995). At each stage birds are subject to a

53 number of disturbances including habitat loss, collisions with wind turbines and cell 
54 phone towers, predation by house cats, exposure to disease, and the increasing effects of

55 global climate change (Altizer et al. 2011; Jonzen et al. 2006; Loss et al. 2013).

56 However, without the ability to connect populations across the annual cycle it is difficult

57 to assess the impact of local stressors on population declines. Historically, efforts to map

58 songbird migration patterns relied on recovery of individual birds previously captured

59 and tagged with bird bands; however, this approach has met with limited success for

60 small-bodied songbirds because recapture rates of birds away from their original banding

61 sites are often very low (< 1 in 10,000) (Faaborg et al. 2010b; Gustafson \& Hildenbrand

62 1999). More recently, geo-locators, small tracking devices that record information on

63 ambient light levels to estimate an individuals location, have increased our knowledge of

64 the migratory pathways in many songbird species (Stutchbury et al. 2009), but remain

65 impractical for most large-scale applications (1000's of individuals) due to cost, weight

66 restrictions, and the need to recover individuals to collect data from the devices (Arlt et

67 al. 2013; Bridge et al. 2013). Alternatively, genetic and isotopic markers that use

68 information contained within the feathers to pinpoint an individuals population of origin

69 have broad appeal because they are cost-effective, noninvasive, and do not require

70 recapture (Kelly et al. 2005; Rubenstein et al. 2002; Rundel et al. 2013), but have been

71 plagued in the past by low resolution and/or technical issues related to working with

72 feathers (Lovette et al. 2004; Segelbacher 2002; Wunder et al. 2005). Thus, there

73 remains a need for a broadly applicable tracking method that can be used to resolve

74 populations on spatial scales that are informative for assessing drivers of regional

75 population declines. 
77 In the last several years, genome sequencing has revolutionized the field of molecular

78 ecology, resulting in new technologies that can be applied to molecular tagging of wild

79 populations (Davey et al. 2011). Genome reduction techniques, such as Restriction Site

80 Associated DNA sequencing (RAD-seq), can be used to sequence multiple individuals

81 across a large fraction of the genome and identify hundreds of thousands of genetic

82 markers that are useful for distinguishing populations (Baird et al. 2008). One type of

83 genetic marker that can be identified from genomic sequence data is a Single Nucleotide

84 Polymorphism (SNP), DNA sequence variation occurring when a single nucleotide in the

85 genetic code - A, T, C, or G - differs between individuals or homologous chromosomes.

86 In particular, SNPs found within or linked to genes under selection often display elevated

87 allele frequencies and, as a result, can be targeted to reveal population structure at finer

88 spatial scales than is possible using neutral genetic markers (Nielsen et al. 2012; Nielsen

89 et al. 2009). Furthermore, SNP-specific assays designed to target small fragments of

90 sequence around the SNP loci of interest can be advantageous in cases where the DNA is

91 highly fragmented or available only in very small quantities, such as DNA from a single,

92 small passerine feather.

94 Here we develop high-resolution SNP markers for tracking populations of a migratory

95 bird, the Wilson's warbler, Cardellina pusilla, using a combination of Restriction Site

96 Associated DNA paired end sequencing (RAD-PE seq) and high throughput SNPtype ${ }^{\mathrm{TM}}$

97 Assay screening. The Wilson's warbler, a long-distance neotropical migratory bird with

98 a cross-continental breeding distribution (Ammon \& Gilbert 1999), is particularly

99 appropriate as model for testing the efficacy of high-resolution molecular markers 
100 because previous population genetic/connectivity studies on this species provide a solid

101 basis for comparison between methods (Clegg et al. 2003; Irwin et al. 2011; Kimura et

102 al. 2002; Paxton et al. 2007; Paxton et al. 2013; Rundel et al. 2013; Yong et al. 1998).

103 By harnessing recent advances in Next-Generation Sequencing we scan the genomes of

104 Wilson's warblers sampled from across the breeding range and identify a set of highly

105 divergent SNP loci with strong potential for population identification. We then develop

106 SNPtype ${ }^{\mathrm{TM}}$ Assays that target these highly divergent loci and use them to screen 1626

107 feather and blood samples collected from across the annual cycle in collaboration with

108 bird banding stations located across North and Central America. We illustrate how the

109 resulting region-specific migration map can be used to help identify drivers of regional

110 demographic trends and inform studies of migrant stopover ecology.

\section{Methods}

113 (a) Sample collection

114 Collection of 1648 feather and blood samples (22 samples for the SNP ascertainment

115 panel and 1626 for the SNP screening panel) from 68 locations across the breeding,

116 wintering and migratory range was made possible through a large collaborative effort

117 with bird banding stations within and outside of the Monitoring Avian Productivity and

118 Survivorship (MAPS), the Landbird Monitoring of North America (LaMNA), and the

119 Monitoreo de Sobrevivencia Invernal (MoSI) networks (Table 1). Genetic samples,

120 consisting of the tip of one outer rectrix or blood collected by brachial vein puncture and

121 preserved in lysis buffer (Seutin 1991), were purified using Qiagen DNeasy Blood and

122 Tissue Kit and quantified using a NanoDrop ${ }^{\mathrm{TM}}$ Spectrophotometer (Thermo Scientific, 
123 Inc) (Smith et al. 2003). Breeding (June 10 - July 31), migratory (March 1 - May 31),

124 and wintering (December 1 - February 28) samples were collected and categorized into

125 groups based on collection date, signs of breeding (presence/size of a cloacal

126 protuberance), signs migration (extent of fat) and life history timetables for the Wilson's

127 warbler (Ammon \& Gilbert 1999). To assess migratory stopover site use through time,

128686 of the 1648 samples from a stopover site located on the Lower Lower Colorado

129 River, near the town Cibola, AZ, were collected using consistent effort, daily, passive

130 mist-netting from March 22 - May 24, across the years 2008 and 2009 (Table 1).

132 (b) SNP discovery

133 To identify SNPs useful for distinguishing genetically distinct regions across the breeding

134 range of the Wilson's warbler, an ascertainment panel of 22 individuals was selected to

135 represent the range of phylogenetic variation known in the species, including all 3

136 recognized subspecies (Ammon \& Gilbert 1999; Kimura et al. 2002). Five individuals

137 from each of five regions were included in the ascertainment panel, except for from the

138 Southwestern region where samples were limited to 2 individuals (SI Table 1). Purified

139 extractions from blood samples were quantified using Quant-iT ${ }^{\mathrm{TM}}$ PicoGreen ${ }^{\circledR} \mathrm{dsDNA}$

140 Assay Kit (Invitrogen Inc), and Restriction Site Associated DNA Paired-end (RAD-PE)

141 libraries containing individually barcoded samples were prepared at Floragenex, Inc.

142 according to Baird et al. (2008) and Ruegg et. al. (Ruegg et al. 2014) (SI Methods).

143 RAD-PE sequencing made it possible to build longer contigs ( $300 \mathrm{bp})$ from short read,

144 100bp Illumina HiSeq2000 data in order to improve downstream bioinformatics and 
145 provide adequate flanking sequence around SNPs for assay development (Etter et al.

146 2011).

148 Samples from each isolate were sequenced on an Illumina HiSeq2000 (Illumina, San

149 Diego, CA) using paired-end 100 bp sequencing reads. Paired-end sequences from each

150 sample were collected, separated by individual, stripped of barcodes, trimmed to $70 \mathrm{bp}$,

151 scrubbed of putative contaminant and high-copy-number-sequences and filtered to

152 include only those with a Phred score $\geq 10$. The sample with the greatest number of reads

153 passing the initial quality filter was used to create a reference set of RAD-PE contigs

154 against which sequences from other samples were aligned. To create the reference,

155 primary reads were clustered into unique RAD markers and the paired-end sequences

156 associated with each RAD tag were assembled de novo using Velvet (Zerbino \& Birney

157 2008) into contigs ranging from $180-610 \mathrm{bp}$, with an average length of $300 \mathrm{bp}$. Paired-

158 end reads from the remaining samples were aligned to this reference using Bowtie

159 (Langmead \& Salzberg 2012) and SNPs were identified using the SAMtools software (Li

160 et al. 2009) with mpileup module under standard conditions.

162 To narrow our dataset to SNPs we could confidently use to assess population structure we

163 performed a second round of quality filtering and removed: (1) putative SNPs with no

164 variants and / or more than two alleles; (2) genotypes in individuals with a quality score

165 of $<30$; (3) genotypes with $<8$ reads in a homozygote or $<4$ reads per allele in

166 heterozygotes; (4) putative SNPs that had suitable genotypes in $<12$ out of the 17

167 samples from four western populations or $<5$ out of the 5 samples from the eastern 
168 population and (5) putative SNPs with $<40$ bp of flanking sequence on either side. To

169 limit the chances of including linked markers genomic coordinates were attained by

170 mapping the remaining contigs to the closest, best annotated, songbird genome, the zebra

171 finch (Taeniopygia guttata) (Version 3.2.4; (Warren et al. 2010)) using BLAST+

172 (version 2.2.25).

173

174 To avoid the possibility of erroneous matches, the data was filtered to include only

175 contigs that aligned to the zebra finch genome with only a single hit and an E-value $<10^{-}$

$17{ }^{40}$. Because SNPs with large frequency differences are the most effective for identifying

177 populations, all SNPs that passed our second round of quality filters were ranked

178 according to frequency differences between the 5 regions (SI Table 2) and 150 SNPs

179 displaying the largest allele frequency differences between each of the 10 pairwise

180 comparisons were selected for conversion to SNPtype ${ }^{\mathrm{TM}}$ Assays (Fluidigm Inc). Before

181 making a final selection, we also considered factors such as: GC content $(<65 \%)$, number

182 of genotypes per population, and average coverage at a SNP across all populations (SI

183 Table 2). An initial assay pre-screening panel was then performed and the assay pool

184 was further reduced to the 96 assays (the number that fit on a single 96.96 Fluidigm

185 Array) that could be genotyped most reliably (SI Table 2).

\section{7 (b) SNP Screening}

188 The Fluidigm Corporation EP1 ${ }^{\mathrm{TM}}$ Genotyping System was used to genotype 96 SNP loci

189 using 94 individuals per run and 2 non-template controls. To avoid the potential for high-

190 grading bias (i.e. wrongly inflating the apparent resolving power of a group of loci for 
191 population identification) (Anderson 2010), none of the 22 samples used in our original

192 ascertainment panel were included in the final SNP screening and population structure

193 analyses. To ensure amplification of low quality or low concentration DNA from

194 feathers, an initial pre-amplification step was performed according to the manufacturers

195 protocol using a primer pool containing 96 unlabeled locus-specific SNPtype primers (SI

196 Methods). PCR products were diluted 1:100 and re-amplified using fluorescently labeled

197 allele-specific primers. The results were imaged on an EP1 Array Reader and alleles

198 were called using Fluidigm's automated Genotyping Analysis Software (Fluidigm Inc)

199 with a confidence threshold of $90 \%$. In addition, all SNP calls were visually inspected

200 and any calls that did not fall clearly into one of three clusters - heterozygote or either

201 homozygote cluster - were removed from the analysis. As DNA quality can affect call

202 accuracy, a stringent quality filter was employed and samples with >90 of 96 missing loci

203 were dropped. To assess the reliability of SNPtype assays for genotyping DNA from a

204 variety of sources (blood and feather extractions), the proportion of samples yielding

205 useable genotype data was calculated. Tests for linkage disequilibrium and conformance

206 to Hardy-Weinberg equilibrium (HWE) (Louis \& Dempster 1987) were performed using

207 GENEPOP software, vers. 4.0 (Rousset 2008).

209 (c) Population structure analysis

210 While genetic differentiation $\left(\mathrm{F}_{\mathrm{ST}}\right)$ is likely inflated because selected loci were not a

211 random sample from the genome, we calculated $\mathrm{F}_{\mathrm{ST}}$ here for comparison to previous

212 genetic analysis. $\mathrm{F}_{\mathrm{ST}}$ between all pairs of populations was calculated as $\theta$ (Weir \&

213 Cockerham 1984), using the software GENETIX vers. 4.05 (Belkhir et al. 1996-2004) 
214 and the data were permuted 1000 times to determine significance. We used the program

215 STRUCTURE ver. 2.2, to further assess the potential for population structure across the

216 breeding grounds (Pritchard et al. 2000). Ten runs at each $K$ value (K=1-9) were

217 performed under the admixture model with correlated allele frequencies using a burn-in

218 period of 50,000 iterations, a run length of 150,000. All scripts used for the

219 STRUCTURE runs and subsequent population genomic analyses are located at

220 https://github.com/eriqande/wiwa-popgen. To simplify comparison of results, the

221 program CLUMPP (Jakobsson \& Rosenberg 2007) was used to reorder the cluster labels

222 between runs, and individual $q$ values (proportion of ancestry inferred from each

223 population within an individual) were plotted using the program Distruct (Rosenberg

224 2004). Visual inspection of Distruct plots allowed identification of regions where

225 geographic barriers to gene flow exist and/or where admixture is likely.

227 To identify how population structure was distributed across geographic space, we used

228 the program GENELAND (Guillot et al. 2005). Analyses in GENELAND were

229 performed under the spatial model assuming uncorrelated allele frequencies. Inference of

230 population structuring was based on 10 independent runs, each allowing the number of

231 populations to vary between 1 and 10. Each run consisted of 2.2 million MCMC

232 iterations with a thinning interval of 100 . Of the 22,000 iterations retained for the MCMC

233 sample after thinning, the first 5,000 were discarded as burn-in. Post processing of the

234 MCMC sample was done upon a 250 by 250 point grid that covered the breeding range of

235 the species. Posterior probability of group membership estimates from GENELAND

236 were visualized as transparency levels of different colors overlaid upon a base map from 
237 Natural Earth (naturalearthdata.com) and clipped to the Wilson's warbler breeding range

238 using a shapefile (NatureServe 2012), making use of the packages sp, rgdal, and raster in

239 R (Bivand et al. 2014; Hijmans 2014; Pebesma \& Bivand 2005; Team 2014) (see

240 https://github.com/eriqande/wiwa-popgen). Thus, within each distinguishable group the

241 transparency of colors is scaled so that the highest posterior probability of membership in

242 the group according to GENELAND is opaque and the smallest is entirely transparent.

244 To assess the accuracy of our baseline for identification of individuals from each

245 population to genetically distinct breeding groups we used the program GSI_Sim

246 (Anderson 2010; Anderson et al. 2008). GSI_Sim uses an unbiased leave-one-out cross-

247 validation method to assess the accuracy of self-assignment of individuals to populations.

248 Posterior probabilities were obtained in GSI_Sim by summing the posterior probabilities

249 of the populations within each genetically distinct group and assigning the individual to

250 the genetically distinct group with the highest posterior probability.

\section{Results}

253 (a) SNP discovery

254 RAD-PE sequencing on 22 individuals from 5 geographic regions representative of the

255 range of phylogenetic variation known in the species resulted in 123,005 contigs (average

256 length $\sim 300 \mathrm{bp}$ ), containing 449,596 SNPs passing our initial quality filters (SI Table 1).

257 The median depth of sequencing across all contigs within a library was $33 \mathrm{x}$ and the

258 average Phred quality score per library was 35 (SI Table 1). Overall, 166,268 SNPs

259 passed the second round of quality filters and 19,707 of those were candidates for 
260 conversion into SNPtype ${ }^{\mathrm{TM}}$ Assays based upon the absence of variation in 40 base pairs

261 of flanking sequence surrounding the SNPs. Candidate SNPs were ranked according to

262 frequency differences, GC content, the number of genotypes per region, and the average

263 coverage and the final panel was composed of 96 SNPs with pairwise frequency

264 differences between regions ranging from $1-0.4$ (SI Table 2). For contigs that could be

265 mapped to the zebra finch genome with high confidence, the minimum distance between

266 SNPs was 41KB and no two SNPs were selected from the same contig in order to avoid

267 the possibility of linked markers (SI Table 2). In this study we refer to the final panel of

26896 highly differentiated SNPs as high-resolution genetic markers.

$270 \quad$ (b) SNP screening

271 The resulting high resolution genetic markers were used to screen 1626 samples collected

272 from 68 sampling locations across the breeding, wintering and migratory range (Table 1),

273 with 117 samples excluded due to low quality genotypes (>6 loci excluded). The

274 samples with the highest proportion of reliable genotypes were from fresh feather

275 extractions $\left(\mathrm{n}_{\text {reliable }} /\right.$ total $=660 / 686$ or $96 \%$ reliable), followed by fresh blood extractions

$276\left(\mathrm{n}_{\text {reliable }} /\right.$ total $=100 / 106$ or $94 \%$ reliable $)$, and finally extractions that were $>3$ years old

$277\left(\mathrm{n}_{\text {reliable }} /\right.$ total $=701 / 786$ or $90 \%$ reliable $)$. Tests for conformity to HWE revealed that all

278 but 1 of the 94 loci (AB_AK_20) in 2 of the 23 breeding populations (D an L; Table 1,

279 Fig. 1b) were in HWE after accounting for multiple comparisons $(\mathrm{p}<0.0005)$. Deviations

280 from HWE were likely the result of small sample sizes and or the unintentional inclusion

281 of late arriving migrants en route to northern breeding sites. No loci were found to be in

282 linkage disequilibrium after accounting for multiple comparisons $(\mathrm{p}<0.0005)$, suggesting 
283 that loci were not physically linked even in cases where zebra finch genome coordinates

284 could not be attained.

286 (c) Population Structure analysis

287 An analysis of population genetic structure on the breeding grounds identified 6

288 genetically distinguishable groups: Alaska (purple, A - D), eastern North America (red,

289 U-W), the Southern Rockies and Colorado Plateau (orange, S, T), the Pacific Northwest

290 (green, G- J), Sierra Nevada (pink, N-P), and Coastal California (yellow, K-M) (Fig. 1a

$291 \& \mathrm{~b}) . \quad$ Pairwise $\mathrm{F}_{\mathrm{ST}}$ 's between groups ranged from $0-0.68$ with an overall $\mathrm{F}_{\mathrm{ST}}$ of 0.179

292 (95\% CI: $0.144-0.218)$. The strongest genetic differentiation was observed between

293 eastern and western groups $\left(\mathrm{F}_{\mathrm{ST}}=0.41-0.68\right)$ with strong genetic differentiation also

294 seen between the Southern Rockies and Colorado Plateau and all other groups $\left(\mathrm{F}_{\mathrm{ST}}=0.09\right.$

$295-0.27$; SI Table 3). The number of genetically distinct groups was set at 6 based upon

296 convergence between results from STRUCTURE ( $k=6$, average $\ln \mathrm{P}(\mathrm{X} \mid \mathrm{K})=-33359)$,

297 GENELAND, and GSI_Sim (Fig. 1a\&b; Table 2). While 7 genetically distinct groups

298 was also strongly supported by GENELAND and STRUCTURE (K=7, average ln P(X|K)

$299=-33286$; SI Fig. 1), with sampling locations from British Columbia and Alberta (E and

300 F) forming a seventh group distinct from Alaska, the power to accurately assign

301 individuals to groups at k=7 decreased significantly using both STRUCTURE and

302 GSI_Sim (SI Fig. 1).

303

304 Leave-one-out cross validation using GSI_Sim indicated that the ability to correctly

305 assign individuals to groups was high, ranging from 80 - 100\%. The eastern group had 
306 the highest probability of correct assignment (100\%), followed by Alaska to Alberta

307 (94\%), the Southern Rockies and Colorado Plateau (92\%), the Pacific Northwest (84\%),

308 the Sierra Nevada (81\%) and Coastal California (80\%) (Fig. 1b; Table 2). The majority

309 of the incorrect assignments were between the Pacific Northwest, Sierra Nevada and

310 Coastal California. Subsequent assignment of migrant and wintering individuals to

311 genetically distinct breeding groups using GSI_sim indicated that Coastal California,

312 Sierra, and Pacific Northwest breeders winter in western Mexico and southern Baja, and

313 migrate north along the Pacific Flyway, with Coastal California and Sierra breeders

314 found to the west of the Lower Colorado River (Fig. 1b; SI Table 4). In contrast,

315 Southern Rocky and Colorado Plateau breeders winter from El Salvador to Costa Rica,

316 and migrate north through the central US, while eastern breeders winter primarily in the

317 Yucatan and southern Costa Rica and migrate north through eastern Texas and New York

318 (Fig. 1b; SI Table 4). Unlike the presence of strong connectivity across much of the

319 range, Wilson's warblers breeding from Alaska to Alberta were identified in all but one

320 of our migratory stopover sites and across all wintering areas, apart from western Mexico

321 and southern Baja (Fig. 1b, all but location g; SI Table 4).

323 Assignment of migrants collected in a time series from Cibola, AZ revealed a strong

324 temporal pattern in stopover site use across the spring migratory period (Fig. 1c; Table 3).

325 Birds en route to coastal California arrived first (week of March 22), followed by birds en

326 route to the Pacific Northwest (week of March 29), the Sierra Nevada (week of Apri 1 5),

327 and Alaska to Alberta (week of April 26). Only a few individuals migrating through the

328 stopover site were identified as Sierra Nevada breeders (3 per year), while no populations 
329 breeding in the Southern Rocky and Colorado Plateau and Eastern U.S. were identified

330 migrating through the stopover site. Temporal patterns in the arrival of spring migrants

331 were replicated across both the years 2008 and 2009 and were consistent regardless of

332 known differences in migration patterns by age and sex (Yong et al. 1998).

\section{Discussion}

335 Full life cycle conservation of declining migrant songbirds has been hindered by lack of

336 an efficient tracking technology that is both broadly applicable and high resolution. Here

337 we demonstrate how high-resolution molecular markers can be applied towards full life

338 cycle conservation of a migrant songbird, the Wilson's warbler, with a degree of

339 reliability and efficiency that has not been demonstrated using previous tracking methods.

340 By harnessing recent advances in Next-Generation Sequencing we show that 96 highly

341 divergent SNPs selected from a large pool of candidates ( 450,000 SNPs) can be used to

342 identify genetically distinct groups on spatial scales that are informative for regional

343 conservation planning. Our analysis indicates that the power to identify individuals to

344 breeding populations is high $(80-100 \%)$ and that reliable genotypes can be attained from

$34596 \%$ of feathers collected non-invasively from established bird monitoring stations across

346 North and Central America. Because of the biallelic nature of the SNPs in our panel, our

347 genetic data are also easier to validate and standardize across labs than isotope and other

348 genetic methods and, once the assays have been developed, it is possible to genotype

$349 \sim 300$ birds per day for $<\$ 10.00 /$ individual in almost any well-equipped molecular

350 laboratory. Overall, the resolution, efficiency, and cost, combined with the ease of

351 feather collection in collaboration with existing bird monitoring/banding infrastructure, 
352 makes high-resolution genetic markers a broadly applicable method for widespread

353 monitoring of declining songbird species.

355 One of the central challenges in migratory bird conservation is that population declines

356 and conservation planning often occur at regional spatial scales, but our knowledge of

357 migratory connections is usually limited to species-wide range maps. For example, in the

358 Wilson's warbler, an analysis of Breeding Bird Survey (BBS) data for the years 1966 -

3592012 suggests that the species is only slightly declining across it's range (BBS Trend = -

$3601.88,95 \% \mathrm{CI}=2.97,-1.11)$, but an analysis of regional trends suggest that populations in

361 the Sierra Nevada and the Southern Rockies/Colorado Plateau are declining more

362 strongly $\left(\right.$ BBS Trend sierra $=-4.71,95 \% \mathrm{CI}=-6.41,-2.85 ;$ BBS Trend $_{\text {rockies }}=-2.95,95 \%$

$363 \mathrm{CI}=-4.32,-1.42$ ) (Sauer et al. 2012). Here we illustrate that by targeting highly

364 divergent SNP loci we can confidently identify a minimum of six genetically distinct

365 groups across the breeding range with a resolution in the western US equivalent to the

366 spatial scale of regional population declines. Furthermore, the spatial scale of our genetic

367 groups is commensurate with many a priori defined Bird Conservation Regions,

368 ecologically distinct areas in North America with similar habitats and resource

369 management issues (Millard et al. 2012). The ability to align the spatial scale of

370 population genetic structure with the spatial scale of population declines and conservation

371 planning provides a powerful framework from which to base full life cycle conservation

372 (Fig. 1a \& b).

373 
374 The Wilson's warbler has been the focus of numerous population genetic/connectivity

375 studies in the past decade (Clegg et al. 2003; Irwin et al. 2011; Kimura et al. 2002;

376 Paxton et al. 2007; Paxton et al. 2013; Rundel et al. 2013; Yong et al. 1998), but none

377 have yielded the depth and clarity of information on migratory connections documented

378 herein. Our results confirm the presence of previously identified connections between

379 birds breeding in Coastal California and wintering in Southern Baja, MX and between

380 birds breeding in eastern North America and wintering in the Yucatan, Belize and Costa

381 Rica (Kimura et al. 2002; Rundel et al. 2013), but also reveal new patterns across time

382 and space that are much richer and stronger then previously recognized. For example,

383 here we show that Wilson's warblers breeding in Coastal California (Fig. 1b, yellow)

384 share their wintering area in southern Baja with Pacific Northwest breeders (Fig. 1b,

385 green) and that both of these groups also winter to the east of Baja in Sinaloa, MX, with

386 Sierra Nevada breeders (Fig. 1b, pink) (Sauer et al. 2012). Samples collected from across

387 the spring migratory period indicate that western breeders from all three groups (Coastal

388 California, Pacific Northwest, and Sierra Nevada) migrate north along the Pacific

389 Flyway, with Coastal California and Sierra Nevada breeders found west of the Lower

390 Colorado River. In addition, we show for the first time that breeders from the Southern

391 Rocky Mountains and Colorado Plateau (Fig. 1b, orange) occupy a restricted El

392 Salvador-to-Costa Rica wintering distribution and migrate North along the Central

393 Flyway, while eastern breeders (Fig. 1b, red) migrate North through eastern Texas and

394 New York. Overall our results indicate that screening high volumes of individuals using

395 high resolution molecular markers can yield a level of clarity in migratory connections 
396 across time and space that has not been previously demonstrated using other tracking

397 techniques.

399 The resulting map for the Wilson's warbler provides an example of how information on 400 region-specific migration patterns can be combined with information on region-specific

401 population declines in order to strengthen predictions about where migrants are most

402 limited. In the case of the Wilson's warbler, BBS data suggests that Sierra Nevada

403 breeders are experiencing strong population declines $(\mathrm{BBS}$ Trend $\mathrm{sierra}=4.71,95 \% \mathrm{CI}=$ -

404 6.41, -2.85), while Pacific Northwest and Coastal California breeders are declining less

405 severely or remaining stable $\left(\right.$ BBS Trend Pacific_Northwest $_{\text {- }}=-1.96,95 \% \mathrm{CI}=-2.54,-1.31$;

406 BBS Trend Coastal_California $\left._{2}=-0.49, \mathrm{CI}=-1.62,0.84\right)$. The fact that all three groups occupy

407 distinct breeding ranges, but mix on their wintering grounds and at migratory stopover

408 sites suggests that declines in Sierra Nevada breeders are likely driven by factors on the

409 breeding grounds. Alternatively, the migration map as a whole suggests that bottlenecks

410 for Wilson's warblers likely occur in areas where multiple genetically distinct breeding

411 groups funnel through the same stopover site or wintering area such as in Coastal

412 California, Western Mexico, and Costa Rica. These results are supported by work in

413 other taxa and further emphasize the importance of stopover habitat for migrant

414 conservation (Sheehy et al. 2011).

416 Migratory passerines spend roughly a quarter of their year en route between breeding and

417 wintering areas, but relatively little is known about the biology and behavior of migrants

418 during the migratory phase of their annual cycle (Faaborg et al. 2010b). The availability 
419 and quality of habitat at stopover sites could have significant effects on populations, but

420 determining the extent to which physiological and ecological demands experienced

421 during migration may limit populations is often contingent upon knowledge of an

422 individuals ultimate destination (Faaborg et al. 2010a; Faaborg et al. 2010b). Here we

423 successfully genotype 609 samples collected in a time series from a stopover site near

424 Cibola, AZ and demonstrate how high-resolution genetic markers can be used to identify

425 the ultimate destination of birds captured en route to their breeding grounds (Fig. 1b \&c;

426 location b). Breaking down the results by week revealed distinct waves of migrants, with

427 Coastal California breeders arriving first (March 22 - 29), followed by Pacific Northwest

428 and Sierra Nevada breeders (March 29-April 5), and Alaska-to-Alberta breeders arriving

429 significantly later (April 19-26). These patterns were replicated across two years and are

430 consistent regardless of known differences in migration patterns by age and sex (Yong et

431 al. 1998). While differences in the timing of migration in Wilson's warblers have been

432 suggested in the past based upon changes in the frequency of haplotypes or isotopic

433 signatures (Paxton et al. 2007; Paxton et al. 2013), this is the first time that anyone has

434 attained individual-level assignments of large numbers of migrants collected in a time

435 series, bringing a new level of clarity to our understanding of stopover site use through

436 time. It is important to note, that the depth of sampling across time that we are able to

437 achieve using high-resolution genetic markers would not have been possible using

438 extrinsic tracking devices, such as geolocators, due of cost and weight restrictions and the

439 need to recapture individuals to collect the information (Arlt et al. 2013; Bridge et al.

440 2013). The resulting information on migratory connections across time can be used to

441 help build timetables of migration along the Pacific Flyway and help to inform when 
442 particularly vulnerable populations may be migrating through an area. Furthermore,

443 because DNA can be collected from all birds, dead or alive, high resolution genetic

444 markers could be used to identify migrants subject to collisions with wind turbines, cell

445 phone towers and other manmade structures.

447 While our results suggest that high-resolution molecular markers surpass previous genetic

448 markers in terms efficiency and resolution, our conclusions could be further strengthened

449 by the inclusion of additional data and analyses. For example, the robustness of the

450 patterns described here varies depending upon the sample size at each location and in

451 some locations, such as in Belize and many of the migratory stopover sites (Fig. 1b,

452 locations $1, \mathrm{~d}, \mathrm{e}, \mathrm{f}, \mathrm{g})$, additional sampling across time and space is needed. In addition,

453 while our assignment probabilities are very high for an intrinsic marker $(80-100 \%)$ there

454 is a potential for incorrect assignments, particularly between the three western groups

455 (Coastal California, Pacific Northwest, and the Sierras) were admixture is likely (Table

456 2). Similarly, there are large regions on the breeding grounds that could not be

457 distinguished using our markers, such as birds breeding from Alberta to Alaska (purple,

458 Fig. 1b). In the future, the addition of more genetic loci as well as the addition of

459 isotopic markers and statistical methods for combining both sources of data into a single

460 statistical framework will help further resolve populations across the range (Rundel et al.

461 2013). Lastly, it is important to note that the spatially explicit depiction of the genetic

462 results generated in GENELAND may not accurately identify the location of boundary

463 between genetic groups. Additional sampling across the projected boundaries will help

464 clarify the location of the genetic breaks as well as the factors driving differences 
465 between Wilson's warblers in each region. Such genetic differences are particularly

466 interesting in light of the documented differences in migratory timing for Wilson's

467 warblers described herein and the potential for migration timing to contribute to

468 divergence in migratory birds more generally (Bearhop et al. 2005; Ruegg et al. 2014;

469 Ruegg et al. 2012).

470

471 A review article by Faaborg et al (Faaborg et al. 2010b) recently identified continuing

472 research needs for Neotropical migrant birds, including identifying migratory pathways

473 and wintering locations, bottlenecks for conservation, and timetables for migration. Here

474 we demonstrate how high-resolution genetic markers designed for Wilson's warblers, can

475 be applied to help address many of these continuing research needs with a level of

476 efficiency and reliability that has not previously been demonstrated. In the last several

477 years there has been a revolution in sequencing technology that has increased by orders

478 of magnitude the amount of sequence data that can be generated, while at the same time

479 reducing the cost of individual-level analysis (Metzker 2010). Our results show that by

480 harnessing recent advances in sequencing technology it is now possible to develop high-

481 resolution genetic markers for tracking populations of migrants on a broad scale. The

482 resulting information on fine-scale population genetic structure, region-specific migratory

483 connections, and timetables of migration provides a powerful framework from which to

484 base full life cycle conservation of declining songbird species. 


\section{Literature Cited}

Altizer S, Bartel R, Han BA (2011) Animal migration and infectious disease risk. Science 331, 296-302.

Ammon E, M., Gilbert W, M. (1999) Wilson's Warbler (Cardellina pusilla). In: The Birds of North America Online (ed. Poole A), Ithaca: Cornell Lab of Ornithology.

Anderson EC (2010) Assessing the power of informative subsets of loci for population assignment: standard methods are upwardly biased. Mol Ecol Resour 10, 701-710.

Anderson EC, Waples RS, Kalinowski ST (2008) An improved method for predicting the accuracy of genetic stock identification. Canadian Journal of Fisheries and Aquatic Sciences 65, 1475-1486.

Arlt D, Low M, Part T (2013) Effect of geolocators on migration and subsequent breeding performance of a long-distance passerine migrant. PLOS ONE 8, e82316.

Baird NA, Etter PD, Atwood TS, et al. (2008) Rapid SNP Discovery and Genetic Mapping Using Sequenced RAD Markers. PLoS ONE 3, e3376.

Bearhop S, Fiedler W, Furness RW, et al. (2005) Assortative mating as a mechanism for rapid evolution of a migratory divide. Science 310, 502-504.

Belkhir K, Borsa P, Chikhi L, Raufaste N, Bonhomme F (1996-2004) GENETIX 4.05, logiciel sous WindowsTM pour le genetique does populations. . Universite de Montpellier II, Montepellier (France), Laboratoire Genome, Populations, Interactions.

Bivand R, Keitt T, Rowlingson B (2014) rgdal: Bindings for the Geospatial Data Abstraction Library. R package version 0.8 - 16.

Bridge ES, Kelly JF, Contina A, et al. (2013) Advances in tracking small migratory birds: a technical review of light-level geolocation. Journal of Field Ornithology 84, 121-137.

Clegg SM, Kelly JF, Kimura M, Smith Thomas B (2003) Combining genetic markers and stable isotopes to reveal population connectivity and migration patterns in a Neotropical migrant, Wilson's warbler (Wilsonia pusilla). Molecular Ecology 12, 819-830.

Davey JW, Hohenlohe PA, Etter PD, et al. (2011) Genome-wide genetic marker discovery and genotyping using next-generation sequencing. Nature Reviews Genetics 12, 499-510.

Etter PD, Preston JL, Bassham S, Cresko WA, Johnson EA (2011) Local De Novo Assembly of RAD Paired-End Contigs Using Short Sequencing Reads. PLoS ONE 6, e18561.

Faaborg J, Holmes RT, Anders AD, et al. (2010a) Recent advances in understanding migration systems of New World land birds. Ecological Monographs 80, 3-48.

Faaborg J, Holmes RT, Anders AD, et al. (2010b) Conserving migratory land birds in the new world: do we know enough? Ecological Applications 20, 398-418.

Guillot G, Estoup A, Mortier F, Cosson JF (2005) A spatial statistical model for landscape genetics. Genetics 170, 1261-1280.

Gustafson ME, Hildenbrand J (1999) Bird Banding Laboratory Homepage, ver 07-102005.

Hijmans RJ (2014) Raster: Geographic data analysis and modeling. R package version 2.2-31. 
Irwin DE, Irwin JH, Smith TB (2011) Genetic variation and seasonal migratory connectivity in Wilson's warblers (Wilsonia pusilla): species-level differences in nuclear DNA between western and eastern populations. Molecular Ecology 20, 3102-3115.

Jakobsson M, Rosenberg NA (2007) CLUMPP: a cluster matching and permutation program for dealing with label switching and multimodality in analysis of population structure. Bioinformatics 23, 1801-1806.

Jonzen N, Linden A, Ergon T, et al. (2006) Rapid advance of spring arrival dates in longdistance migratory birds. Science 312, 1959-1961.

Kelly JF, Ruegg KC, Smith TB (2005) Combining isotopic and genetic markers to identify breeding origins of migrant birds. Ecological Applications 15, 14871497.

Kimura M, Clegg SM, Lovette IJ, et al. (2002) Phylogeographical approaches to assessing demographic connectivity between breeding and overwintering regions in a Nearctic-Neotropical warbler (Wilsonia pusilla). Molecular Ecology 11, 1605-1616.

Langmead B, Salzberg SL (2012) Fast gapped-read alignment with Bowtie 2. Nat Methods 9, 357-359.

Li H, Handsaker B, Wysoker A, et al. (2009) The Sequence Alignment/Map format and SAMtools. Bioinformatics 25, 2078-2079.

Loss SR, Will T, Marra PP (2013) The impact of free-ranging domestic cats on wildlife of the United States. Nat Commun 4, 1396.

Louis EJ, Dempster ER (1987) An exact test for Hardy-Weinberg and multiple alleles. Biometrics 43, 805-811.

Lovette IJ, Clegg SM, Smith TB (2004) Limited Utility of mtDNA Markers for Determining Connectivity among Breeding and Overwintering Locations in Three Neotropical Migrant Birds. Conservation Biology 18, 156-166.

Metzker ML (2010) Sequencing technologies - the next generation. Nature Reviews Genetics 11, 31-46.

Millard MJ, Czarnecki CA, Morton JM, et al. (2012) A National Geographic Framework for Guiding Conservation on a Landscape Scale. Journal of Fish and Wildlife Management 3, 175-183.

NatureServe BIa (2012) Bird species distribution maps of the world NatureServe, Arlington, USA.

Nielsen EE, Cariani A, Mac Aoidh E, et al. (2012) Gene-associated markers provide tools for tackling illegal fishing and false eco-certification. Nat Commun 3, 851.

Nielsen EE, Hemmer-Hansen J, Larsen PF, Bekkevold D (2009) Population genomics of marine fishes: identifying adaptive variation in space and time. Molecular Ecology 18, 3128-3150.

Paxton KL, Van Ripper III C, Theimer TC, Paxton EH (2007) Spatial and temporal migration patterns of Wilson's warbler (Wilsonia pusilla) in the southeast as revealed by stable isotopes. The Auk 124, 162-175.

Paxton KL, Yau M, Moore FR, Irwin D (2013) Differential migratory timing of western populations of wilson's warblers (Cardellina pusilla) revealed by mitochondiral DNA and stable isotopes. Auk 130, 689.

Pebesma EJ, Bivand RS (2005) Classes and methods for spatial data in R. R News 5. 
Pritchard JK, Stephens M, Donnelly P (2000) Inference of population structure using multilocus genotype data. Genetics 155, 945-959.

Rappole JH (1995) The ecology of migrant birds - a Neotropical perspective Smithsonian Institution Press, Washington.

Robbins CS, J. R. Sauer, R. Greenberg, and S. Droege (1989) Population declines in North American birds that migrate to the Neotropics. Proc. Nat. Acad. Sci. 86, 7658-7662.

Rosenberg NA (2004) Distruct: a program for the graphical display of population structure. Molecular Ecology Notes 4.

Rousset F (2008) genepop'007: a complete re-implementation of the genepop software for Windows and Linux. Molecular Ecology Resources 8, 103-106.

Rubenstein DR, Chamberlain CP, Holmes RT, et al. (2002) Linking breeding and wintering ranges of a migratory songbird using stable isotopes. Science $\mathbf{2 9 5}$, 1062-1065.

Ruegg K, Anderson EC, Boone J, Pouls J, Smith TB (2014) A role for migration-linked genes and genomic islands in divergence of a songbird. Molecular Ecology.

Ruegg K, Anderson EC, Slabbekoorn H (2012) Differences in timing of migration and response to sexual signalling drive asymmetric hybridization across a migratory divide. Journal of Evolutionary Biology 25, 1741-1750.

Rundel CW, Wunder MB, Alvarado AH, et al. (2013) Novel statistical methods for integrating genetic and stable isotope data to infer individual-level migratory connectivity. Molecular Ecology 22, 4163-4176.

Sauer JR, Hines JE, Fallon JE, et al. (2012) The North American Breeding Bird Survey, Results and Analysis 1966-2011. USGS Patuxent Wildlife Research Center, Laurel, MD.

Segelbacher G (2002) Noninvasive genetic analysis in birds: testing reliability of feather samples. Molecular Ecology Notes 2, 367-369.

Seutin G, B. N. White and P. T. Boag (1991) Preservation of avian blood and tissue samples for DNA analyses. Canadian Journal of Zoology 69, 82-90.

Sheehy J, Taylor CM, Norris DR (2011) The importance of stopover habitat for developing effective conservation strategies for migratory animals. Journal of Ornithology 152, 161-168.

Smith TB, Wayne RK, Marra PP, et al. (2003) A call for feather sampling. The Auk 120, 218-221.

Stutchbury BJ, Tarof SA, Done T, et al. (2009) Tracking long-distance songbird migration by using geolocators. Science 323, 896 .

Team RC (2014) R: A language and environment for statistical computing., Vienna, Austria. http://www.R-project.org/

Warren WC, Clayton DF, Ellegren H, et al. (2010) The genome of a songbird. Nature 464, 757-762.

Weir BS, Cockerham CC (1984) Estimating F-statistics for the analysis of population structure. Evolution 38, 1358-1370.

Wunder MB, Kester CL, Knopf FL, Rye RO (2005) A test of geographic assignment using isotope tracers in feathers of known origin. Oecologia 144, 607-617.

Yong W, Finch DM, Moore FR, Kelly JF (1998) Stopover ecology and habitat use of migratory Wilson's Warblers. Auk 115, 829-842. 
bioRxiv preprint doi: $\mathrm{https}: / /$ doi.org/10.1101/007757; this version posted August 8,2014 . The copyright holder for this preprint (which was not certified by peer review) is the author/funder. All rights reserved. No reuse allowed without permission.

623 Zerbino DR, Birney E (2008) Velvet: algorithms for de novo short read assembly using 624 de Bruijn graphs. Genome Research 18, 821-829.

625 
626 Acknowledgements. We would like to thank J.C. Garza at the Southwest Fisheries

627 Science Center for the use of laboratory space and equipment. This research was

628 supported by a grant to K. Ruegg from the California Institute for the Energy and the

629 Environment (POEA01-Z01), a donation from Margery Nicolson, a grant to T.B. Smith

630 from The Turner Foundation, EPA (RD-83377801), and a grant to F. Moore from the

631 National Science Foundation (IOS-0844703). We would also like to thank J. Boone and

632 T. Atwood for their assistance with the laboratory and bioinformatics components of the

633 assay design, the many LaMNA, MAPS, and MoSI station operators who contributed

634 avian tissue samples, C.J. Ralph, L. West, D. Kaschube, P. Pyle, J. Saracco, and R.

635 Taylor for coordinating sampling efforts. We are grateful to the USDI Fish and Wildlife

636 Service, the National Park Service, and USDA Forest Service for funding to help operate

637 LaMNA, MAPS and MoSI stations that provided feather samples for this work as well as

638 field crews and staff at Cibola National Wildlife Refuge for help with sample collection

639 and field logistics.

640

641 Author Contributions. K. Ruegg and T.B. Smith conceived of the study and K. Ruegg

642 wrote the majority of the manuscript and conducted and/or oversaw the analyses. E.C.

643 Anderson wrote the scripts for the population genomic analyses and figure creation. K.

644 Paxton and F. Moore contributed ideas and genetic material for the analysis of migrants

645 from Cibola, AZ. V. Apkenas conducted and helped analyze data for the SNP screening.

646 S. Lao assisted with feather sample organization, extraction, and the analysis of

647 genotyping reliability scores. R.B. Siegel and D.F. DeSante facilitated the collection of

648 feather samples in collaboration with bird banding stations within and outside of the 
649 Monitoring Avian Productivity and Survivorship (MAPS) and the Monitoreo de

650 Sobrevivencia Invernal (MoSI) networks.

651

\section{Figure Ledgend}

653 Figure 1. Migratory connections in the Wilson's warbler identified using SNP-based

654 genetic markers. A) Results from STRUCTURE showing 6 genetically distinct

655 populations across the breeding grounds. Capital letters (A-W) refer to the location of

656 breeding populations depicted on the map in B as well as listed in Table 1. B) Spatially

657 explicit population structure across the annual cycle. The colors across the breeding

658 range represent the results from GENELAND which were post-processed using R so that

659 the density of each color relects the relative posterior probability of membership for each

660 pixel to the most probable of the 6 different genetic clusters (see text). The results were

661 clipped to the species distribution map (NatureServe 2012). Lower case letters (a-g)

662 represent the location of wintering and spring migratory samples (Table 1). Pie charts

663 indicate the proportion of wintering indidiviuals assigned to each breeding group with the

664 number of individuals listed at the center of each pie. Arrows represent the proportion of

665 migrants assigned to each breeding group with the numbers of indviduals listed at the top

666 of the arrows. C) The proportion of indivdiuals assigned to each breeding population

667 across spring migration of 2008 and 2009. Numbers in the center of the pies refer to

668 sample sizes and the data are grouped by week with the date representing the mid-week

669 date in a non-leap year. 
Table 1. Number of Wilson's warblers successfully screened at each location across the species breeding, wintering and migratory range. Locations in close proximity were merged on the map in Fig. 1. Uppercase letters are reserved for breeding populations, while lower case letters are reserved for migratory stopover and wintering locations.

Location

Latitude Longitude N Population

Breeding (Jun 10 - July 31)

Cantwell_1, Denali National Park, AK

63.449

$-150.813$

10

A

Cantwell_2, Denali National Park, AK

63.594

$-149.611$

11

A

Denali, Denali National Park, AK

63.716

$-149.088$

8

A

Yakutat, AK

59.514

$-139.681$

21

B

Ugashik_1, AK

57.175

$-157.269$

10

C

Ugashik_2, AK

57.183

$-157.283$

16

C

Juneau, AK

58.300

$-134.400$

10

D

Hardisty Creek, Calgary, AB

53.500

$-117.500$

2

E

Ram Falls, Calgary, AB

52.000

$-115.800$

5

E

Benjamin Creek, Calgary, AB

51.500

$-115.000$

2

E

Beaver Dam, Calgary, AB

51.10

$-114.063$

16

E 
100 Mile House, BC

Darrington, WA

Silverton, WA

Roy, WA

Harlan, OR

McKenzie Bridge, OR

Eureka, CA

Half Moon Bay, CA

Big Sur, CA

San Luis Obispo, CA

Tennant, CA

Clio, CA

Hume, CA

Hillary Meadow, MT

Crow Creek, MT

Elgin_1, OR

Elgin_2, OR
51.700

48.208

48.051

47.056

44.506

44.199

40.783

37.506

36.286

35.195

41.492

39.667

36.799

48.347

47.471

45.817

45.679
$-121.300$

$-121.576$

$-121.433 \quad 5$

$-122.488 \quad 4$

G

$\mathrm{H}$

$-123.630 \quad 23$

22

I

J

K

L

M

$\mathrm{N}$

$-121.939 \quad 25$

$-120.600$

15

$\mathrm{O}$

$-118.599 \quad 16$

$\mathrm{P}$

Q

Q

$\mathrm{R}$

$\mathrm{R}$ 
Pingree Park, Fort Colins, CO

Grand Mesa, CO

Camp Myrica, QC

Hilliardton, ON

Fredericton, NB
40.550

39.000

49.700

47.500

45.800
$-105.567$

$-107.900$

$-73.300$

$-79.700$

$-66.700$
19

11

17

4

4

\section{Migratory Stopover (March - May)}

O'neil Forbay Wildlife Area, CA

Lower Colorado River, Cibola, AZ

Buenos Aires National Wildlife Refuge, AZ

San Pedro Riparian National Cons. Area,

c

Albuquerque, NM

35.013

$$
-106.465
$$

12

d

Sierra del Carmen_1, Coahuila, MX

Sierra del Carmen_2, Coahuila, MX

Fairview, TX

Braddock Bay, NY

$28.909-102.546 \quad 4 \quad$ e

$\begin{array}{llll}28.861 & -102.650 & 3 & \text { e }\end{array}$

$\begin{array}{llll}33.152 & -96.600 \quad 43 \quad f\end{array}$

$\begin{array}{llll}43.161 & -77.611 & 19 & \mathrm{~g}\end{array}$




\section{Wintering (Dec - Feb)}

San Jose del Cabo, Baja California Sur, MX

Chupaderos, Sinaloa, MX

Las Joyas, Autlan, Jalisco, MX

Nevado de Colima, Colima, Jalisco, MX

U. of Mexico, San Angel, Distrito Federal,
22.883

23.333

19.767

19.233

19.313

23.000

19.450

19.548

17.100

18.400

17.094

13.821

13.850

13.943

14.403
$-109.900$

8

$-105.500 \quad 8$

$-104.367 \quad 25$

$-103.717$

3

j

Coatapec, Veracruz, MX

Chaa Creek, San Ignacio, BE

Izalco, Sonsonate, SV

Los Andes National Park, Santa Ana, SV

Las Lajas, Santa Ana, SV

Metapan, Santa Ana, SV
$-99.100$

15

$-96.967$

13

$\mathrm{m}$

$-96.921$

7

$\mathrm{m}$

$-96.800$

14

n

$-95.200$

9

O

$\mathrm{p}$

$-89.653$

17

q

$-89.620$

7

q

$\begin{array}{ll}-89.617 & 7\end{array}$

q

q 


\begin{tabular}{|c|c|c|c|c|}
\hline San Salvador Volcano, SV & 13.700 & -89.200 & 12 & $\mathrm{q}$ \\
\hline Cantoral, Tegucigalpa, HN & 14.331 & -87.399 & 11 & $\mathrm{r}$ \\
\hline La Tigra National Park, Tegucigalpa, HN & 14.100 & -87.217 & 15 & $\mathrm{r}$ \\
\hline El Jaguar Cafetal, Jinotega, NI & 13.229 & -86.053 & 10 & $\mathrm{~s}$ \\
\hline Volcan Mombacho, Granada, NI & 11.832 & -86.008 & 2 & $\mathrm{~s}$ \\
\hline Monteverde Cloud Forest, Santa Elena, CR & 10.314 & -84.825 & 9 & $\mathrm{t}$ \\
\hline San Vito_1, Puntarenas, CR & 8.754 & -82.926 & 2 & $\mathrm{u}$ \\
\hline San Vito_2, Puntarenas, CR & 8.766 & -82.943 & 2 & $\mathrm{u}$ \\
\hline San Vito_3, Puntarenas, CR & 8.784 & -82.975 & 5 & $\mathrm{u}$ \\
\hline San Vito_4, Puntarenaus, CR & 8.809 & -82.924 & 1 & $\mathrm{u}$ \\
\hline San Vito_5, Puntarenaus, CR & 8.822 & -82.972 & 12 & $\mathrm{u}$ \\
\hline
\end{tabular}


Table 2. Assignment of Wilson's warblers of known origin back to breeding population using GSI_Sim. Population names are listed in Table 1 and the colors indicate the genetic group ( Fig. 1).

\begin{tabular}{|c|c|c|c|c|c|c|}
\hline $\begin{array}{c}\text { Population } \\
\text { (Fig. 1, } \\
\text { Table 1) }\end{array}$ & $\begin{array}{c}\text { Alaska to } \\
\text { Alberta }\end{array}$ & $\begin{array}{c}\text { Pacific } \\
\text { Northwest }\end{array}$ & $\begin{array}{c}\text { Coastal } \\
\text { California }\end{array}$ & Sierra & $\begin{array}{c}\text { Rocky } \\
\text { Mountain }\end{array}$ & Eastern \\
\hline A & 29 & 0 & 0 & 0 & 0 & 0 \\
\hline B & 21 & 0 & 0 & 0 & 0 & 0 \\
\hline $\mathrm{C}$ & 26 & 0 & 0 & 0 & 0 & 0 \\
\hline D & 10 & 0 & 0 & 0 & 0 & 0 \\
\hline $\mathbf{E}$ & 24 & 0 & 0 & 0 & 1 & 0 \\
\hline $\mathbf{F}$ & 9 & 0 & 0 & 0 & 4 & 0 \\
\hline $\mathbf{G}$ & 2 & 9 & 1 & 0 & 0 & 0 \\
\hline $\mathbf{H}$ & 0 & 20 & 3 & 0 & 0 & 0 \\
\hline $\mathbf{I}$ & 0 & 20 & 1 & 1 & 0 & 0 \\
\hline $\mathbf{J}$ & 0 & 15 & 0 & 3 & 0 & 0 \\
\hline $\mathbf{K}$ & 0 & 2 & 14 & 1 & 0 & 0 \\
\hline $\mathbf{L}$ & 0 & 3 & 11 & 1 & 0 & 0 \\
\hline $\mathbf{M}$ & 0 & 1 & 19 & 3 & 0 & 0 \\
\hline $\mathbf{N}$ & 0 & 2 & 2 & 21 & 0 & 0 \\
\hline $\mathbf{O}$ & 0 & 1 & 2 & 12 & 0 & 0 \\
\hline $\mathbf{P}$ & 0 & 1 & 0 & 15 & 0 & 0 \\
\hline $\mathbf{Q}$ & 1 & 0 & 0 & 0 & 2 & 0 \\
\hline $\mathbf{R}$ & 6 & 0 & 0 & 0 & 19 & 0 \\
\hline $\mathbf{S}$ & 0 & 0 & 0 & 0 & 19 & 0 \\
\hline $\mathbf{T}$ & 0 & 0 & 0 & 0 & 11 & 0 \\
\hline $\mathbf{U}$ & 0 & 0 & 0 & 0 & 0 & 17 \\
\hline V & 0 & 0 & 0 & 0 & 0 & 4 \\
\hline W & 0 & 0 & 0 & 0 & 0 & 4 \\
\hline
\end{tabular}


Table 3. Genetic identification of Wilson's Warblers migrating through Cibola, CA by week across the years 2008 and 2009. Results represent the individuals assigned to one of the six genetically distinct groups using the program GSI Sim and the data corresponds to the information presented in Figure 1c.

\begin{tabular}{|c|c|c|c|c|c|c|c|}
\hline $\begin{array}{l}\text { Mid-week } \\
\text { Date* }\end{array}$ & Week & $\begin{array}{c}\text { Alaska } \\
\text { to } \\
\text { Alberta }\end{array}$ & $\begin{array}{c}\text { Pacific } \\
\text { NW }\end{array}$ & $\begin{array}{c}\text { Coastal } \\
\text { CA }\end{array}$ & $\begin{array}{c}\text { Sierra } \\
\text { Nevada }\end{array}$ & $\begin{array}{c}\text { Rocky } \\
\text { Mt. }\end{array}$ & Eastern \\
\hline
\end{tabular}

Year 2008

$\begin{array}{lccccccc}\text { 21-Mar } & 11 & 0 & 0 & 0 & 0 & 0 & 0 \\ \text { 28-Mar } & 12 & 0 & 3 & 1 & 0 & 0 & 0 \\ \text { 4-Apr } & 13 & 0 & 11 & 16 & 1 & 0 & 0 \\ \text { 11-Apr } & 14 & 0 & 9 & 4 & 1 & 0 & 0 \\ \text { 18-Apr } & 15 & 0 & 5 & 0 & 0 & 0 & 0 \\ \text { 25-Apr } & 16 & 16 & 11 & 1 & 0 & 0 & 0 \\ \text { 2-May } & 17 & 24 & 6 & 0 & 0 & 0 & 0 \\ \text { 9-May } & 18 & 32 & 2 & 0 & 0 & 0 & 0 \\ \text { 16-May } & 19 & 46 & 3 & 0 & 1 & 0 & 0 \\ \text { 23-May } & 20 & 25 & 0 & 0 & 0 & 0 & 0\end{array}$

Year 2009

$11-0$

2

0

0

0

29-Mar

12

0

3

7

0

0

0

5-Apr

13

0

5

10

0

0

0 


\begin{tabular}{lccccccc} 
12-Apr & 14 & 0 & 6 & 10 & 0 & 0 & 0 \\
19-Apr & 15 & 0 & 10 & 6 & 1 & 0 & 0 \\
26-Apr & 16 & 12 & 6 & 0 & 0 & 0 & 0 \\
3-May & 17 & 74 & 21 & 6 & 1 & 0 & 0 \\
10-May & 18 & 56 & 25 & 1 & 1 & 0 & 0 \\
17-May & 19 & 82 & 6 & 0 & 0 & 0 & 0 \\
24-May & 20 & 33 & 1 & 1 & 0 & 0 & 0 \\
\hline
\end{tabular}

* Dates represent the midweek date in a non-leap year. 
A) Breeding population structure

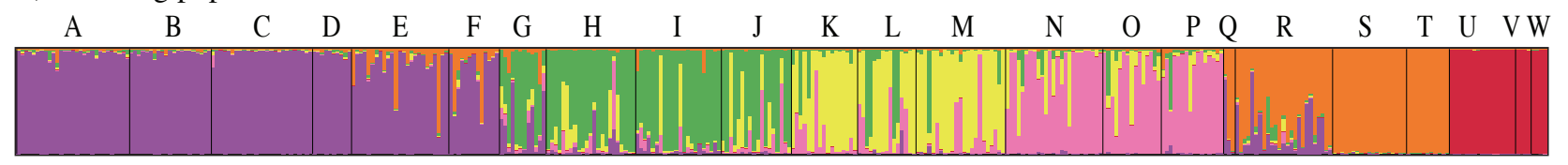

B) Spatially explicit population structure

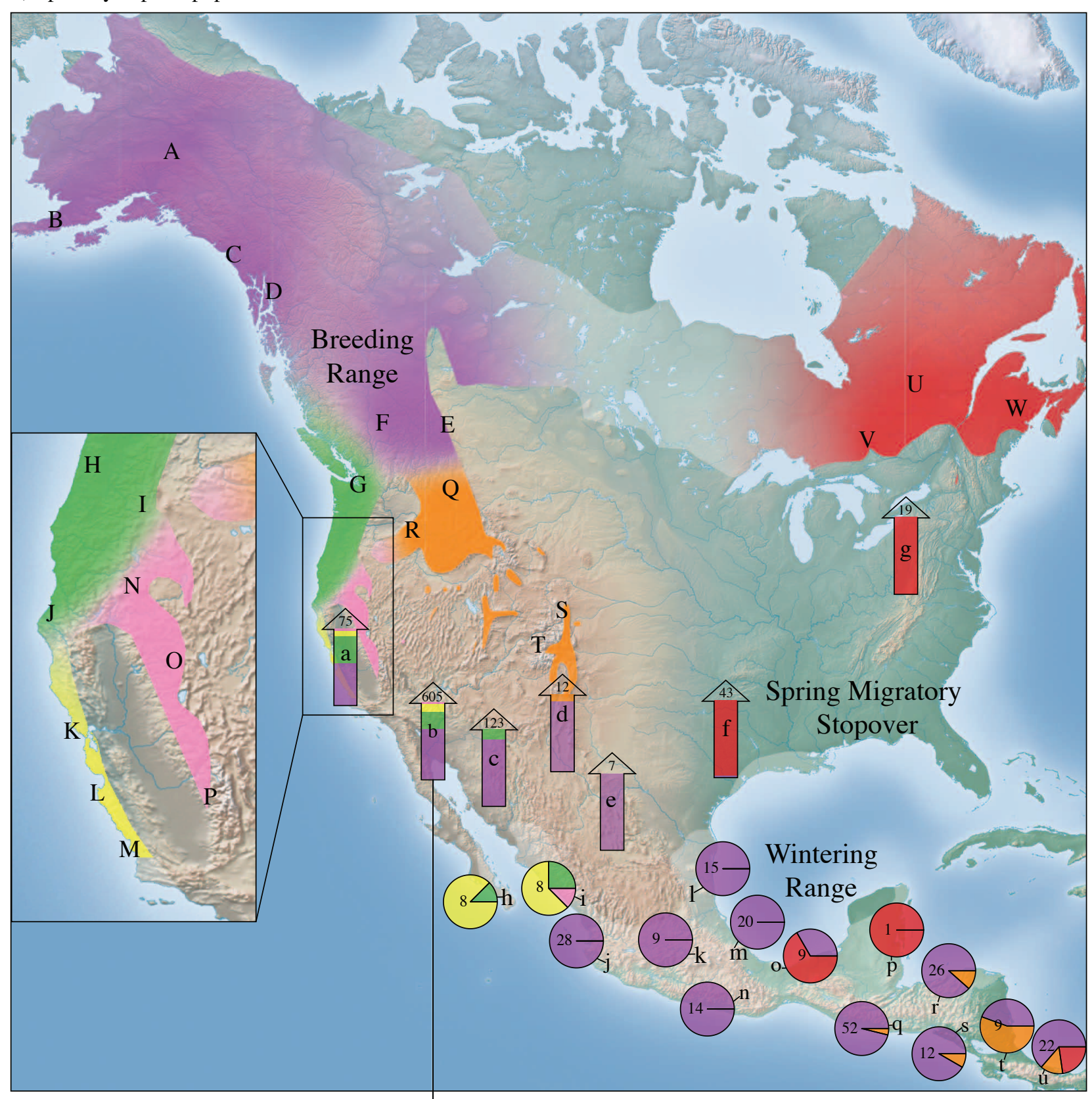

C) Population structure across time, Cibola CA (b, above)

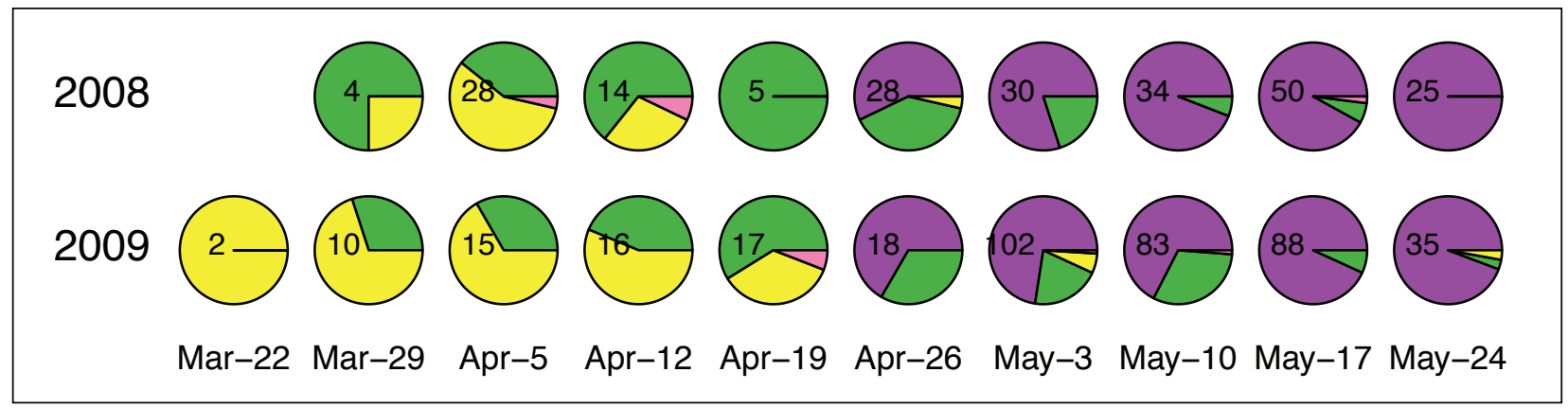

